\title{
Studies of Large-Amplitude $\delta$ Scuti Variables
}

\author{
W. J. F. Wilson, E. F. Milone and D. J. I. Fry \\ Rothney Astrophysical Observatory (RAO) \\ Department of Physics and Astronomy, The University of Calgary
}

The determination of Baade-Wesselink radii and luminosities for pulsating stars are long-standing and highly desired goals since they provide the promise of being standard candles. In a modest contribution towards these goals, we have undertaken a programme to determine the radii and luminosities of the large-amplitude $\delta$ Scuti stars DY Herculis, EH Librae and DY Pegasi from optical and infrared photometry and cross-correlated radial velocity data. We use Fourier representations for $V, I$ and $J$ light curves and for the radial velocity curves in Baade-Wesselink analyses to derive minimum radii over the pulsation cycles. These radii and their errors and the mean bolometric luminosities and absolute magnitudes will be discussed here and in papers to follow. As a check, we also apply our method to the data and results of other groups.

Each $V$ light curve was divided into 200 equally spaced phase points, and a value was calculated for $R_{\min }$ at each phase point in the descending portion of the colour index curve using phase pairs of equal colour index. The error $\delta R_{\min }$ was calculated at each point from the computed errors in the Fourier coefficients. $R_{\min }$ and $\delta R_{\min }$ were then plotted against phase and the mean values $\left\langle R_{\min }\right\rangle$ and $\left\langle\delta R_{\min }\right\rangle$ were calculated over the phase range where $\delta R_{\min }$ was smallest.

We obtain minimum radii from the phase ranges shown of

$$
\begin{array}{ll}
\text { EH Lib }(0.1 \leq \phi \leq 0.5): & <R_{\min }> \pm<\delta R_{\min }>=2.54 \pm 0.30 R_{\odot} \\
\text { DY Her }(-0.03 \leq \phi \leq 0.33): & <R_{\min }> \pm<\delta R_{\min }>=3.26 \pm 0.30 R_{\odot}
\end{array}
$$

For $\mathrm{EH} \mathrm{Lib}$, we combine our values of $R_{\min }+$ computed displacement with the values of $T_{\text {eff }}$ from Joner (1986) to obtain mean values of the bolometric luminosity and absolute bolometric magnitude of

$$
\begin{aligned}
& <L_{\text {bol }}>=23.1(+5.6,-5.1) L_{\odot} \\
& <M_{\text {bol }}>=1.34(-0.24,+0.27) .
\end{aligned}
$$

Fuller descriptions will be published elsewhere. This work has been supported by the Department of Physics and Astronomy, and NSERC grants to EFM.

\section{References:}

Joner, M.D., 1986, PASP 98, 651 